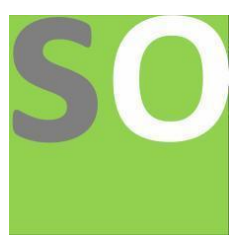

Article title: Parameters for the Tractrix

Authors: Edward Brell[1]

Affiliations: Independant researcher[1]

Orcid ids: 0000-0002-2869-234X[1]

Contact e-mail: drbrell@gmail.com

License information: This work has been published open access under Creative Commons Attribution License http://creativecommons.org/licenses/by/4.0/, which permits unrestricted use, distribution, and reproduction in any medium, provided the original work is properly cited. Conditions, terms of use and publishing policy can be found at https://www.scienceopen.com/.

Preprint statement: This article is a preprint and has not been peer-reviewed, under consideration and submitted to ScienceOpen Preprints for open peer review.

DOI: 10.14293/S2199-1006.1.SOR-.PPWLKVG.v1

Preprint first posted online: 11 September 2020

Keywords: Tractrix, towing, caravan, traler 


\section{Parameters for the Tractrix}

\section{Dr Edward Brell BSc MEng PhD}

The tractrix is a curve which tracks the path of a dragged object. On an X-Y graph it will show the co-ordinates of the object where it has been and where it is going. Such a graph is dimensionless but once a dimension is introduced all other dimensional aspects follow. Whether the object is a recalcitrant dog on a leash, a water skier or a log with one end being dragged along the ground, the path followed will be a tractrix shape, ceteris paribus.

This blog is intended to show how passage of time is introduced to the functioning tractrix.

On a Cartesian plane it can be described by the following transcendental equation:

$$
L \cdot \ln \left(\frac{L+\sqrt{L^{2}-y^{2}}}{y}\right)-\sqrt{L^{2}-y^{2}} \quad \text { Equation } 1
$$

Equation 1 is clumsy, $x$ being a function of $y$. i.e. $x=f(y)$. When Equation 1 is graphed in Figure 1 the following results, showing caricature water skiers.

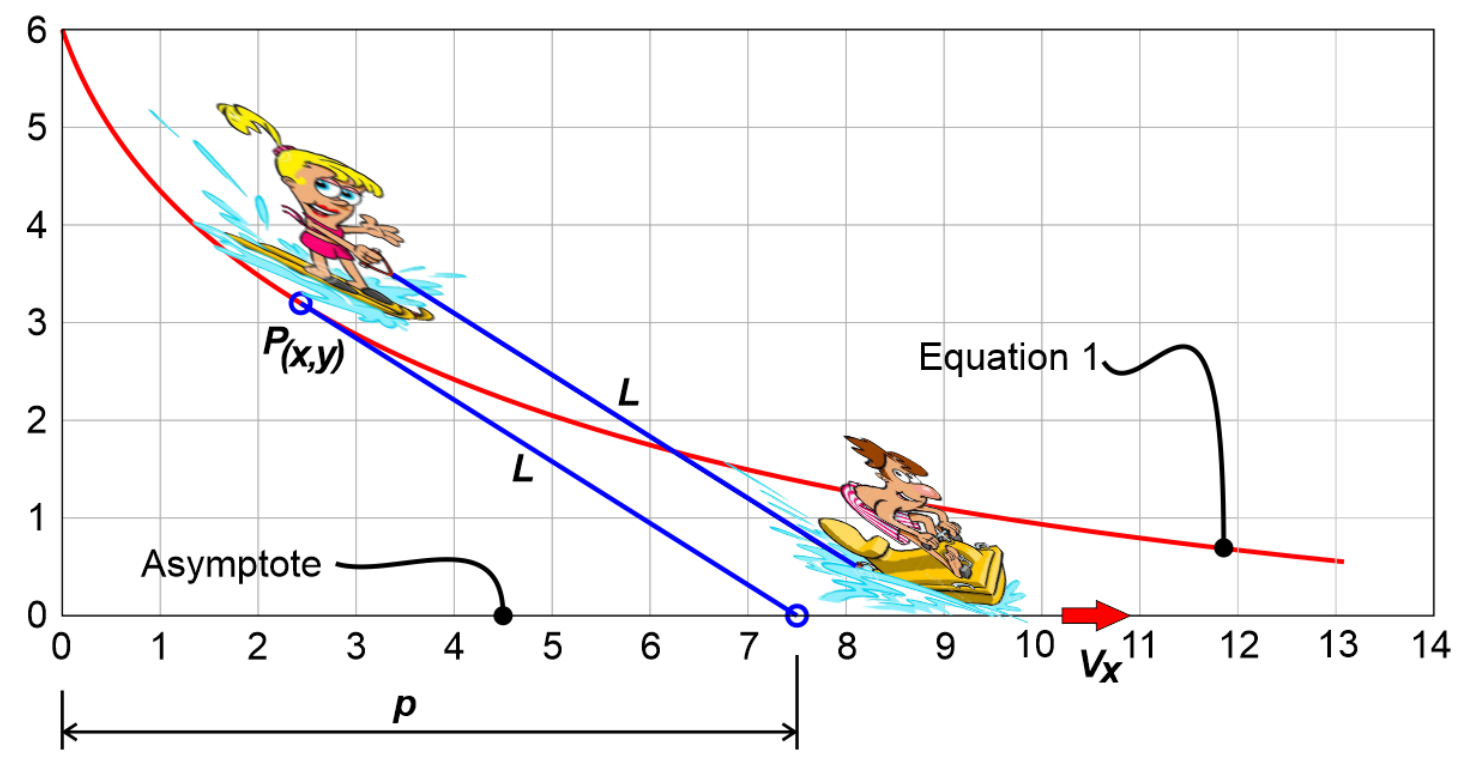

Figure 1 - Tractrix from transcendental equation

Figure 1 is non-dimensional and could represent feet, inches or metres as long as the length of rope or dog leash as long as the units match.

Sreenivasan et al., (2007), offered a parametric equation that can be graphed from two parametric equations, as below:

$$
x(p)=p-L \tanh \left(\frac{p}{L}\right), \quad y(p)=L \operatorname{sech}\left(\frac{p}{L}\right) \quad \text { Equation } 2
$$

Where the parameter $\boldsymbol{p}$ is shown in Figure 1. 


$$
p=x+\sqrt{L^{2}-y^{2}}
$$

Equation 3

There are times where true time $\boldsymbol{t}$ is needed. A useful parameter which makes use of real time $\boldsymbol{t}$ (whether seconds, minutes or days) using real time.

$$
p=\frac{V x * t}{L} \quad \text { Equation } 4
$$

Equation 2 is graphed using the new dimensionless parameter $\mathbf{p}$ for a $6 \mathrm{~m}$ tractrix running on the asymptote at $10 \mathrm{~m} / \mathrm{s}$ for 2 seconds in Figure 2.

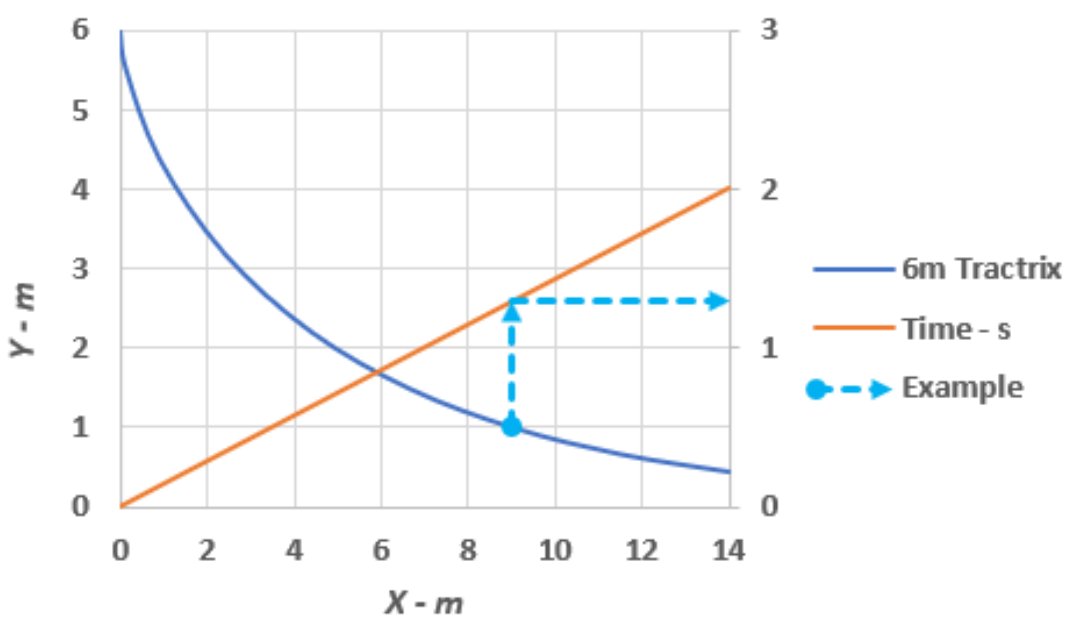

Figure 2-6m Tractrix to Equation 2 using $p$ as a parameter.

The upper regions of the tractrix are of little practical value as clearly $10 \mathrm{~m} / \mathrm{s}$ velocity at say, 0 to 0.5 seconds is untenable. However, there is scope to consider a time-slice at say 1 seconds to 2 seconds. Here the X-distance runs from $7 \mathrm{~m}$ to $14 \mathrm{~m}$ and the $Y$-distance changes from approximately $1.3 \mathrm{~m}$ to $0.4 \mathrm{~m}$ towards the asymptote.

Varying speed of tow vehicle maintains the same tractrix shape. The towed object simply goes further on the same tractrix. A $10 \mathrm{~m}$ tractrix was studied, each for 3 seconds, at speeds of $20,40 \& 60 \mathrm{~km} / \mathrm{h}$. The results were graphed in Figure 3 where all 3 curves were started at zero time. Here the slower speeds are overlaid. 


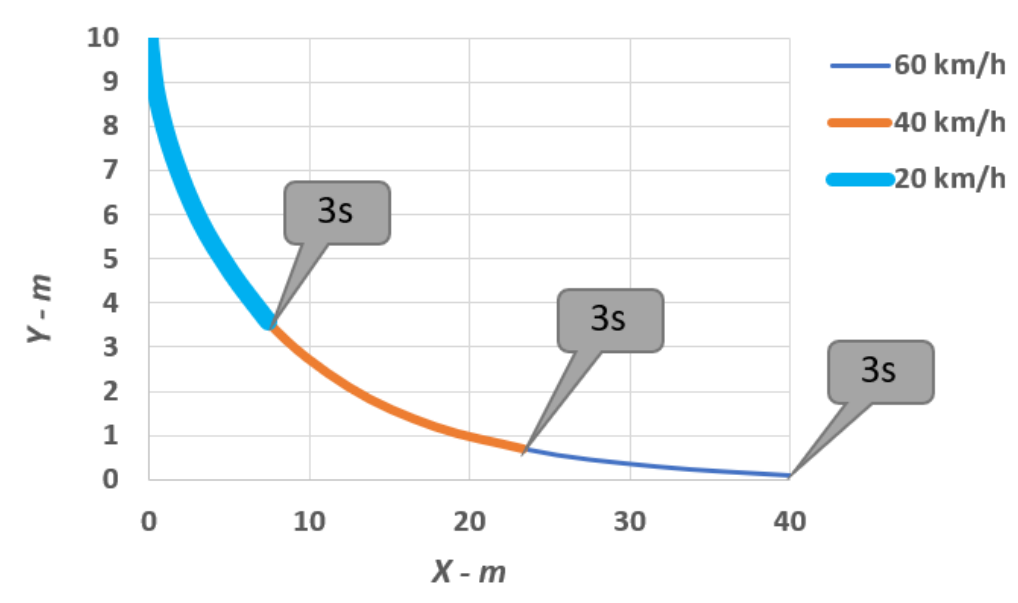

Figure 3-10 m Tractrix to Equation 2 varying speed of tow vehicle

The slope of the taut rope changes as the tow boat progresses. An equation for $\boldsymbol{\theta}$, the slope that the taut rope makes with the asymptote will now be developed. Figure 4 refers.

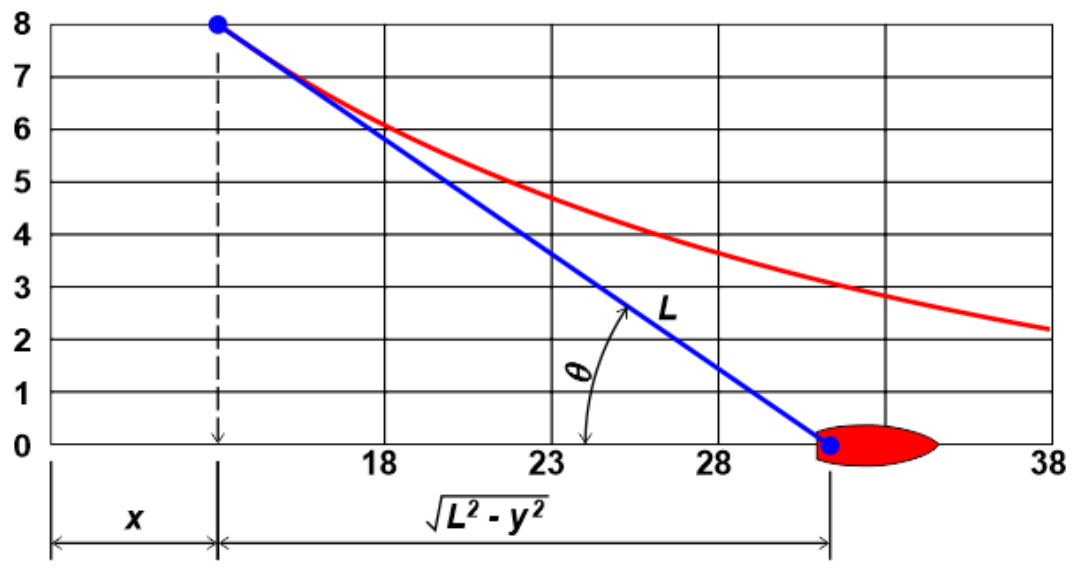

Figure 4-20 m Tractrix to Equation 2 showing angle of rope.

The relationship for $\boldsymbol{\theta}$ can be found from:

$$
\tan (\theta)=\frac{y}{\sqrt{L^{2}-y^{2}}} \quad \text { Equation } 5
$$

Substituting the parametric Equation 2, for $\boldsymbol{y}$ :

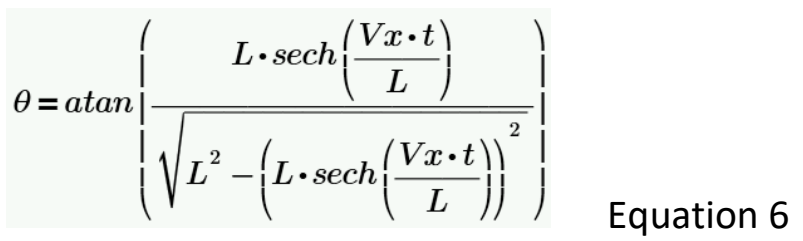

Equation 6 is plotted for the first 5 seconds of travel at speeds of $60,80 \& 100 \mathrm{~km} / \mathrm{h}$ in Figure $\mathbf{5}$ for a $20 \mathrm{~m}$ tractrix. 


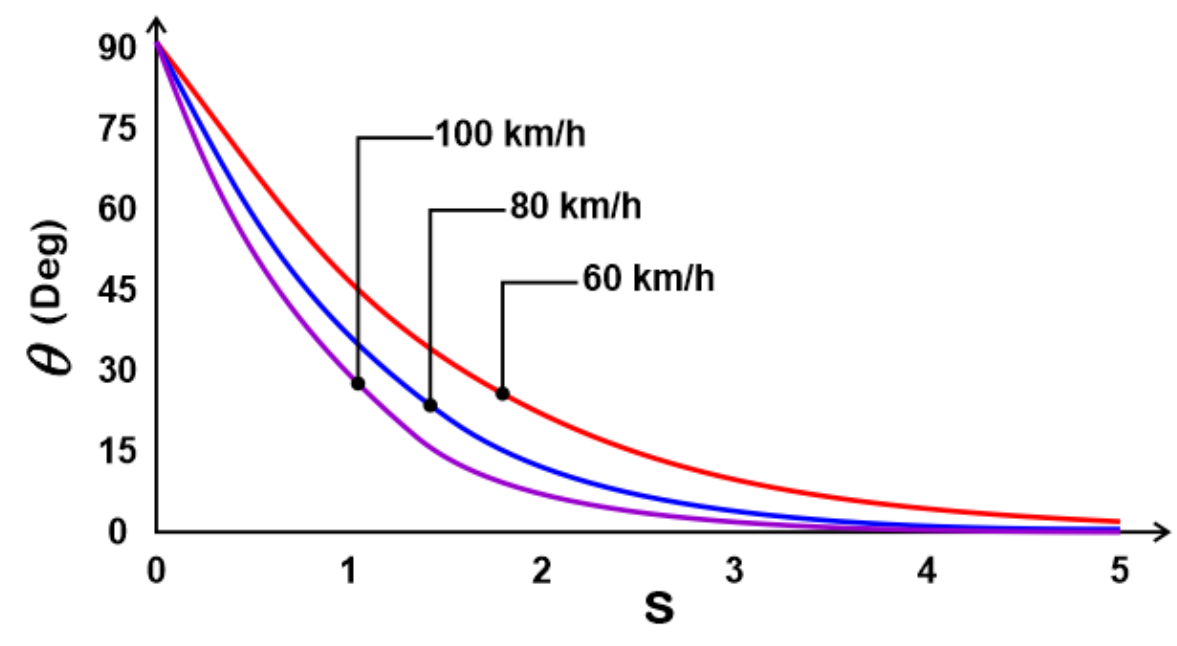

Figure 5 - Angle of tow connection to asymptote for various tow speeds.

Figure 5 shows that greater speed is associated with a more rapid closing of articulation angle in studies like towing trailers and caravans.

There are times in real tractrix systems where the curve starts somewhere other than at zero time. This is illustrated in the case study below. 


\section{CASE STUDY}

Evaporation lowered the level of an artificial lake to a point where a dead tree loomed just below the surface. A water skier at the lake having just negotiated a jump became destabilized by interaction with the invisibly submerged tree. The lake speed limit was 60 $\mathrm{km} / \mathrm{h}$ and a shore video provided critical times. The Coroner wants the exact location of the tree.

\section{Assumptions}

- Tow rope length is $L=20 \mathrm{~m}$.

- Tow boat path is straight and $8 \mathrm{~m}$ from the jump centreline.

- Ski rope remains tangent to tractrix. (as a sack of potatoes would ensure)

- Point of landing after the jump has been calculated prior and is $13 \mathrm{~m}$ from the edge of the jump.

- Tow boat speed is assumed $V x=60 \mathrm{~km} / \mathrm{h}$.

- Reference times from video are shown below:

\section{Calculations}

\section{At Water Landing}

$$
x 1:=L \cdot\left(\frac{V x \cdot 1.88 s}{L}-\tanh \left(\frac{V x \cdot 1.88 s}{L}\right)\right)=13 \mathrm{~m} \quad y 1:=L \cdot \operatorname{sech}\left(\frac{V x \cdot 1.88 s}{L}\right)=8 \mathrm{~m}
$$

\section{At Incident Point}

$$
x 2:=L \cdot\left(\frac{V x \cdot 2.75 s}{L}-\tanh \left(\frac{V x \cdot 2.75 s}{L}\right)\right)=26.2 \mathrm{~m} \quad y 2:=L \cdot \operatorname{sech}\left(\frac{V x \cdot 2.75 s}{L}\right)=4 \mathrm{~m}
$$

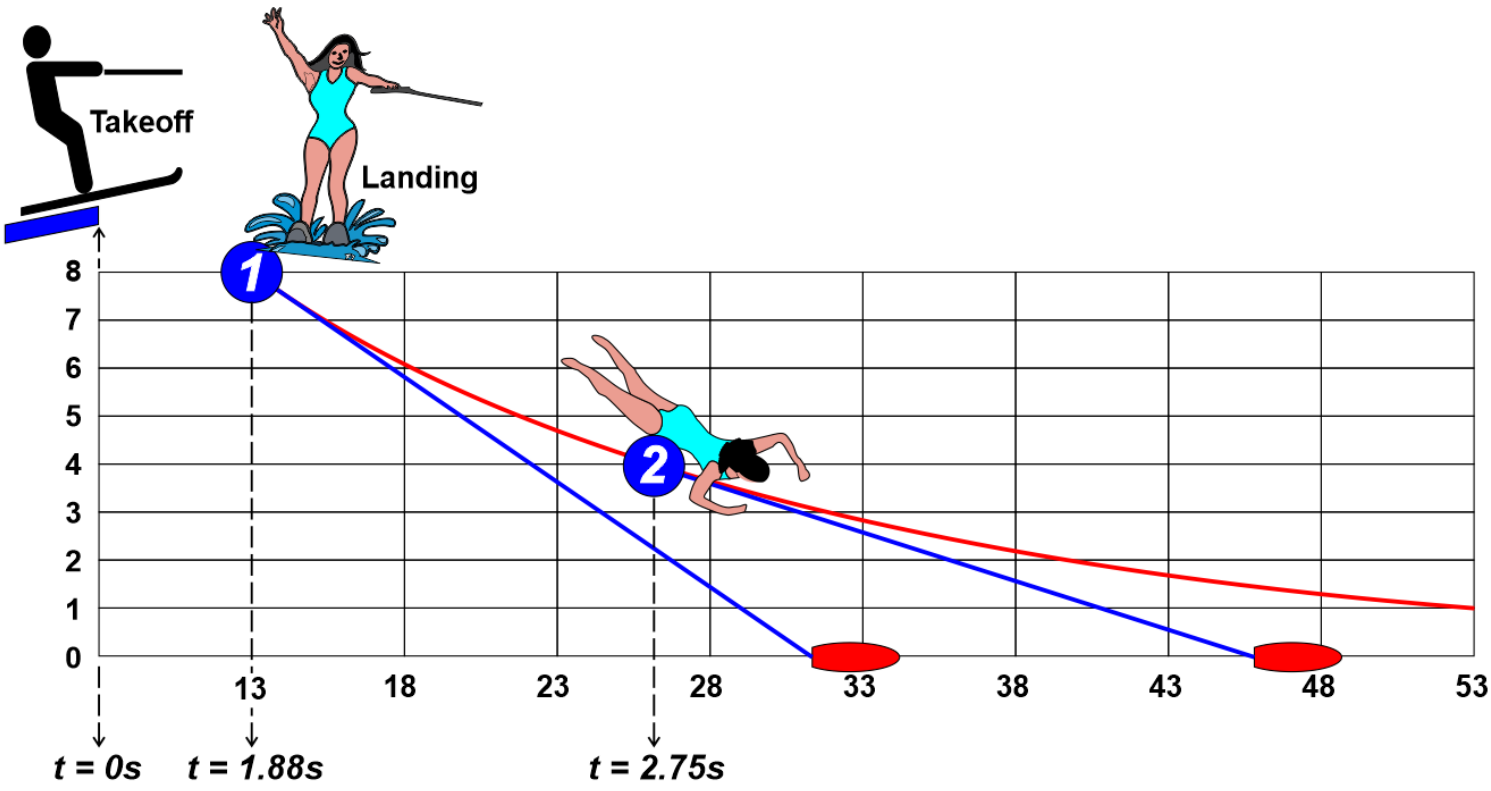

Figure 6-20 m Tractrix started $1.88 \mathrm{~s}$ showing incident at $2.75 \mathrm{~s}$. 


\section{Conclusion}

The incident was calculated to have occurred approximately $4 \mathrm{~m}$ South of the ramp centreline and $26 \mathrm{~m}$ West from the takeoff edge of the ramp.

\section{REFERENCES}

Sreenivasan, S., Goel, P., \& Ghosal, A. (2007). Reduncy resolution using a tractrix. 12th IFToMM World Congresss, 18-21. 